\title{
Caregivers Acceptance of Using Semantic Communication Boards for teaching Children with Complex Communication Needs
}

\author{
Jayr A. Pereira ${ }^{1}$, Jaylton A. Pereira ${ }^{1}$, Robson do N. Fidalgo ${ }^{1}$ \\ ${ }^{1}$ Centro de Informática - Universidade Federal de Pernambuco (UFPE) \\ Recife - PE - Brazil \\ $\{$ jap2, jap3,rdnf\}@cin.ufpe.br
}

\begin{abstract}
Alternative Communication Boards (ACB) are tools used to compensate for the difficulties faced by people with complex communication needs. These boards facilitate the construction of telegraphic phrases through visual cues, using colors and pictograms to represent the grammatical class and the meaning of the words, respectively. In this paper, we present the combination of three essential materials to construct a semantic ACB. In this context, a Semantic ACB is a communication board that uses a semantic script to guide the message authoring. The proposal was evaluated using the Technology Acceptance Model (TAM) as a basis. The results demonstrate that caregivers are more interested in a semantic ACB that is useful than in one that is easy to use.
\end{abstract}

\section{Introduction}

Alternative Communication Boards (ACB) are high-tech Augmentative and Alternative Communication (AAC) systems [ASHA 2021] that try to compensate the difficulties faced by people with complex communication needs (CCN). Generally, these tools are based on cards (a.k.a. pictograms) composed of figures with captions, which configure a sentence when arranged in sequence (cf. Figure 1). Usually, the sentences constructed in the ACBs follow a telegraphic structure, that is, only with keywords (i.e., nouns, verbs, adjectives, and adverbs) and without connecting words (i.e., prepositions, articles, and conjunctions) or verbal conjugations. To facilitate the construction of messages, ACBs such as those illustrated in Figure 1, use visual cues based on colors and pictograms, which give clues about the grammatical class and the meaning of the words, respectively. However, clues that explore the semantic roles of words in sentences tend to be more significant [Bryan 1997, Bolderson et al. 2011]. The work of [Hernández et al. 2014] proposes an approach based on a user-centered recommendation model that guides sentence construction through questions and answers. However, this approach is not based on pictograms and uses long questions, such as "What activity did you do?", "Where did you do it?", and "When did you do it?". Thus, this approach requires skills that people with CCN generally do not retain.

[Bryan 1997] proposed a therapeutic tool called Colourful Semantics (CS), that combines colors and questions like Who?, What Doing? and What? to guide user in sentence construction. CS has the aim of helping children with $\mathrm{CCN}$ to understand and compose well-structured and meaningful sentences in natural language. Numerous studies demonstrate the effectiveness of using CS in the treatment of children with speech difficulties. In the first application made by [Bryan 1997], for example, after 


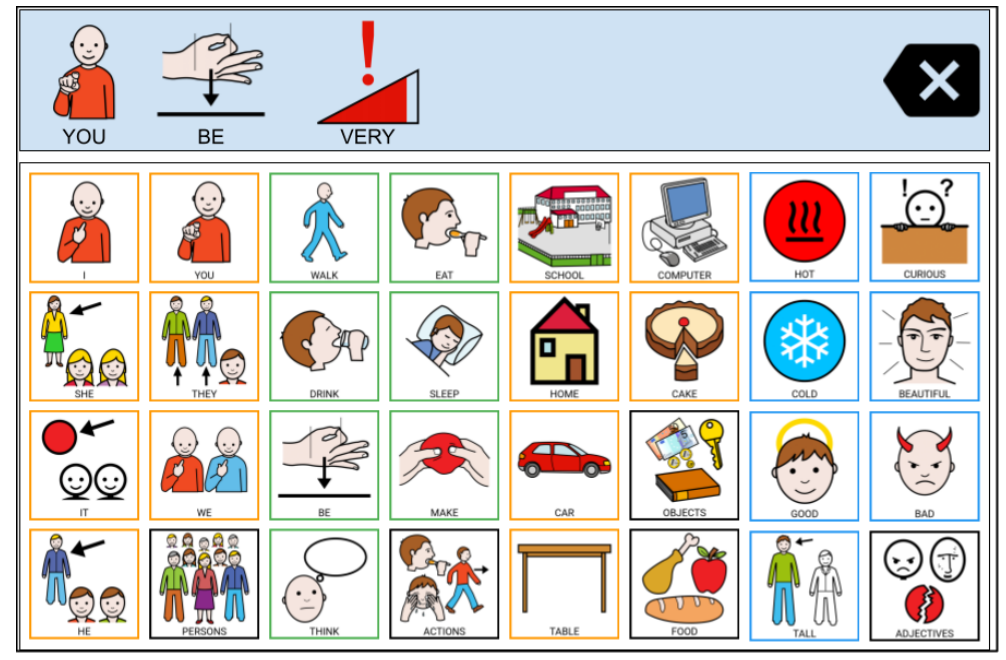

Figure 1. Example of Assistive Communication Board. The board contains a content area (large rectangle at the bottom) with the available cards for selection. The phrase area (blue rectangle at the top) presents the selected cards arranged to form the sentence.

eight weeks of treatment, the child was able to identify and use semantic roles in the construction of phrases during the telling of a story. After a few months, it was also possible to notice an advance in constructing more complex sentences than those initially taught. [Bolderson et al. 2011] and [Christopoulou et al. 2021] also noticed increasing in the communication performance of participants. Despite this, there are no records of AAC systems that implement CS for communication.

In [Pereira et al. 2020], we proposed a Semantic Grammar (SG) based on the CS grammatical structure. This SG can be used as a basis by ACBs to help users in the message composition, allowing visual clues and a semantic script to assist users in finding the more adequate cards to compose telegraphic sentences. This study evaluates the acceptance of professionals who care for children with $\mathrm{CCN}$ about using a semantic ACB as a therapeutic and educational tool. In this context, a Semantic ACB is a communication board that uses a semantic script to guide the message authoring. To accomplish this objective, we developed a prototype of a semantic ACB that combines: 1) Colourful Semantics [Bryan 1997] structure; 2) the Language Acquisition Through Motor Planning (LAMP) approach [Halloran and Halloran 2006]; and 3) the semantic grammar proposed in [Pereira et al. 2020]. The procedure for constructing sentences using the ACB was presented to health and education professionals that were asked to answer a questionnaire based on the Technology Acceptance Model (TAM) [Davis et al. 1989]. We aim to evaluate which of the TAM's essential factors (i.e., perceived usefulness and perceived ease of use) influences the acceptance of the proposed technology. The results suggest that caregivers are more interested in the usefulness of such a tool than its ease of use.

\section{Materials and Methods}

This section presents the material used for building the semantic ACB, the theory related to them, and how we propose combining them. Besides, we cover the Technology Acceptance Model (TAM) and how we used it for evaluating ACB acceptance. 


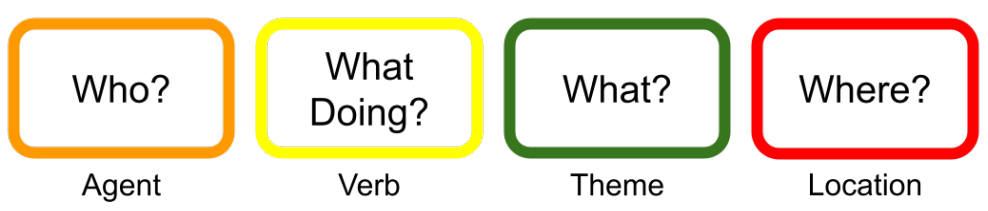

Figure 2. Colourful Semantics

\subsection{Colourful Semantic}

The Colourful Semantics' (CS) [Bryan 1997] initial aim was to serve as a therapeutic tool to help children with CCN to understand and construct sentences through a semantic script that refers to syntactic structures. It can be helpful to support children with a limited vocabulary to organize the grammatical content of their sentences [Christopoulou et al. 2021]. CS's semantic script consists of a system of colors associated with questions (e.g., Who?, What Doing?, What?) that help children understand the semantic role of each sentence's element. According to [Bolderson et al. 2011], the main advantage of CS is identifying the semantic roles of each constituent of a sentence. That is, identifying the function performed by a word concerning the predicate it modifies. For example, in the utterance "The boy eats popcorn", the word "boy" is the agent of the verbal predicate "eats", while "popcorn" is the theme.

Figure 2 illustrates the four basic semantic roles of CS: Agent, Verb, Theme, and Location. These roles are associated with colors in order to: (i) make visual discrimination between each semantic role; (ii) further establish the relationship between the question and the semantic role; (iii) associate each type of phrase with a visual sequence of colors; and (iv) alert the child when he omits a semantic role [Bryan 1997]. Colors act as a visual aid to indicate the grammatical structure of a sentence, and questions help to link this structure (syntactic) to its meaning (semantics) [Law et al. 2012]. Semantic roles are more significant for individuals with $\mathrm{CCN}$ than syntactic functions (i.e., subject, verb, and object) [Bryan 1997]. Its usage is what differentiates CS from other color-coding systems in which the colors refer to grammatical classes (e.g., nouns, verbs, or adjectives).

\subsection{Language Acquisition Through Motor Planning}

Language Acquisition Through Motor Planning (LAMP) [Halloran and Halloran 2006] is based on principles of neurological and motor learning. Its objective is to promote the independence of people with CCN for expressing themselves in any setting. LAMP contains five teaching elements that provide people with $\mathrm{CCN}$ the language skills necessary to learn effective communication. These elements are (1) readiness to learn; (2) joint attention and shared focus; (3) natural consequence; (4) auditory signals; (5) consistent and unique motor patterns [Halloran and Halloran 2006]. According to [Bedwani et al. 2015], the most particular aspect of LAMP is the importance of consistent motor patterns. One can describe these patterns as a hand memory similar to typing or playing the piano. The keys stay in the exact location, and the user's fingers automatically know where to go after a period of practice.

Motor planning can be divided into three phases: cognitive, associative, and automatic learning [Dijksterhuis et al. 2000, Salmoni et al. 1984]. In the cognitive stage, the user learns the target behavior (i.e., communication using the ACB) [Koegel et al. 1999]. 


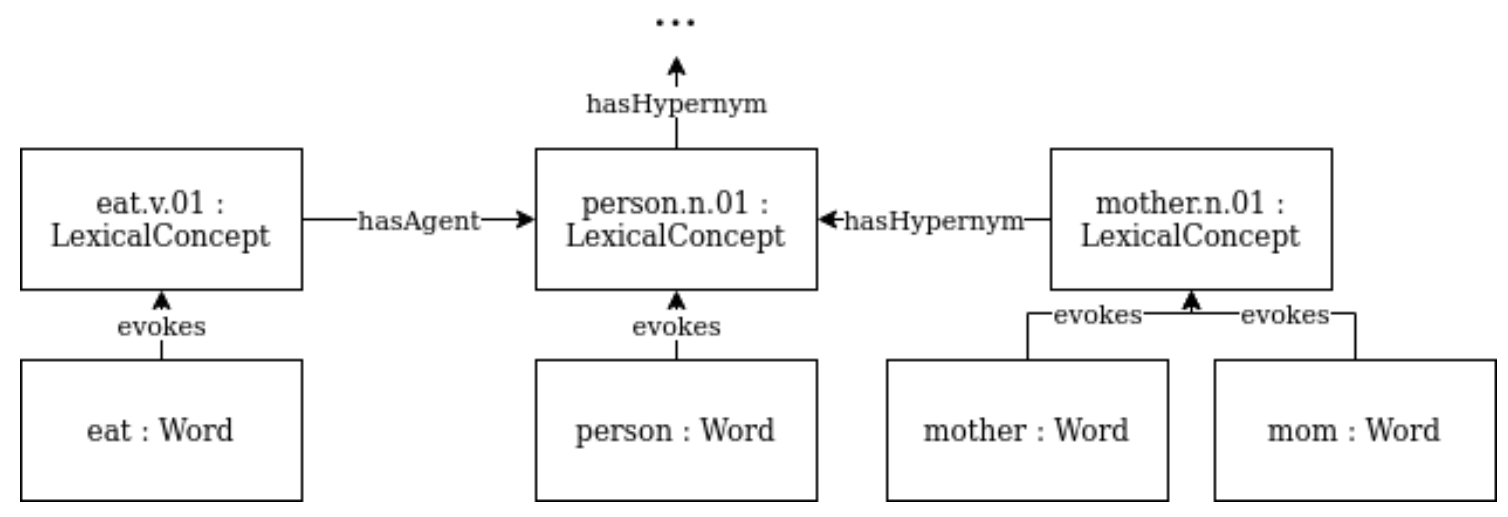

Figure 3. Semantic Grammar Excerpt

In the associative stage, the user practices the learned behavior to move between active thinking and automatic implementation [Ganz et al. 2014, Ganz and Hong 2014]. In the automatic stage, the user no longer needs to think about making the behavior as he/she retained it in his/her memory [Koegel et al. 2003]. This way, by using LAMP, finding a card in an ACB may become automatic behavior after a period of practice.

\subsection{Semantic Grammars}

A Semantic Grammar (SG) [Burton 1976] is a knowledge base in which lexical-semantic properties of hierarchy and predicate-argument connect the concepts evoked by the words of a controlled vocabulary. Hierarchy properties define a semantic structure based on the relationships of hyperonymy and hyponymy between concepts (e.g., the mammal is hyperonym for cat and is hyponym for animal). In comparison, predicate-argument properties define the grammatical structure with the arguments (e.g., agent, theme, attribute) that each word with predicate characteristics (i.e., verbs and nouns) can have. For example, in the sentence "The cat drinks milk" the verbal predicate "drinks" has two arguments: "The cat", as an agent, and "milk", as the theme, which make up the structure agent + verb + theme.

In an SG, the arguments of the grammatical structure are filled in by the semantic structure elements (i.e., concepts), creating relationships like the one illustrated in Figure 3 (eat.v.01 hasAgent person.n.01). This allows the lexical-semantic knowledge of hierarchy (e.g., the cat is an animal) to be used in the process of parsing or generating texts in natural language, avoiding the ambiguity that can exist when using only grammatical classes (e.g., noun) [Burton 1976]. Figure 3 presents an excerpt of the SG proposed in [Pereira et al. 2020], in which each word in the controlled vocabulary evokes a lexical concept, which carries its meaning. Lexical concepts are organized into two taxonomies: one for verbs and one for nouns. Verb taxonomy nodes (e.g., eat.v.01) contain predicate-argument properties (e.g., hasAgent) that point to noun taxonomy nodes (e.g., person.n.01). The predicate-argument properties are based on the CS semantic roles (cf. Section 2.1), and the relationships between concepts (e.g., eat.v.01 has Agent person.n.01) were automaticly extracted from text samples. 
(A)

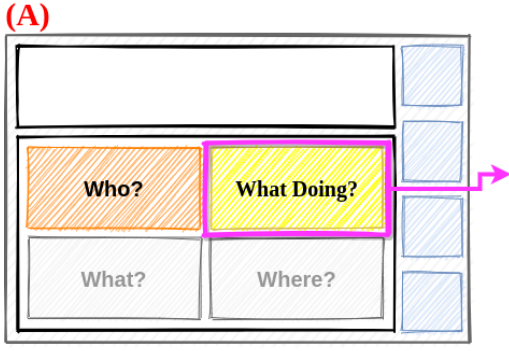

(D)

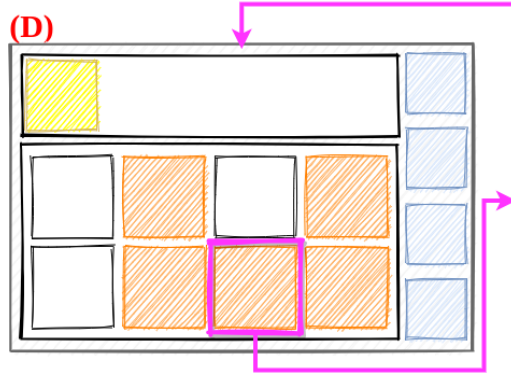

(G)

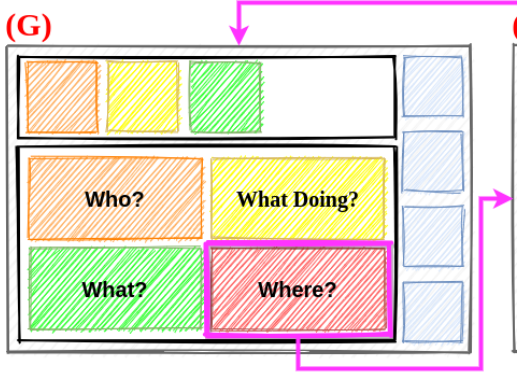

(B)
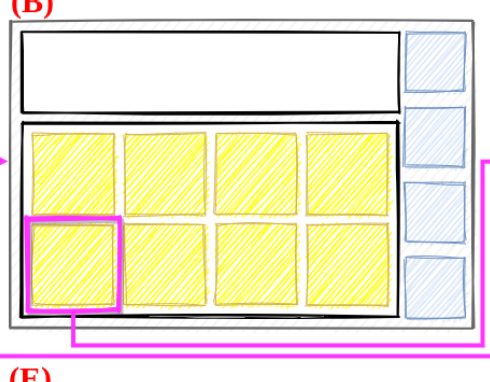

(E)

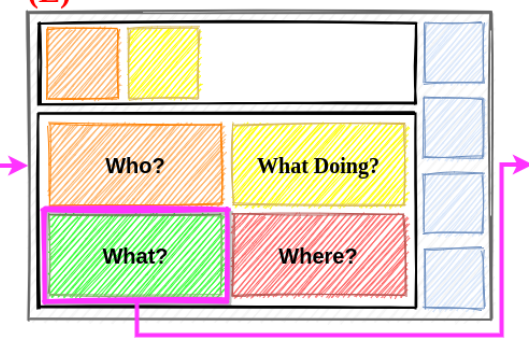

(H)

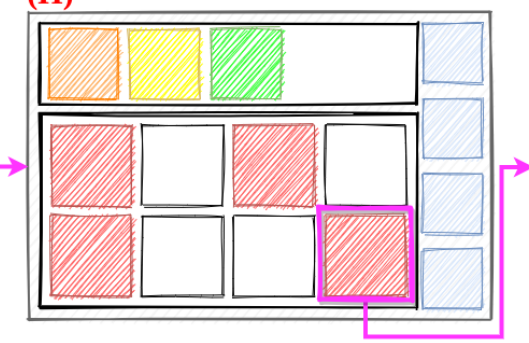

(C)

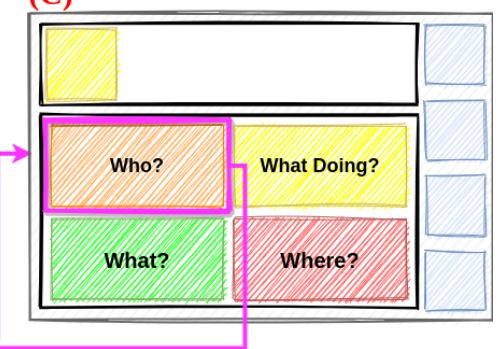

(F)

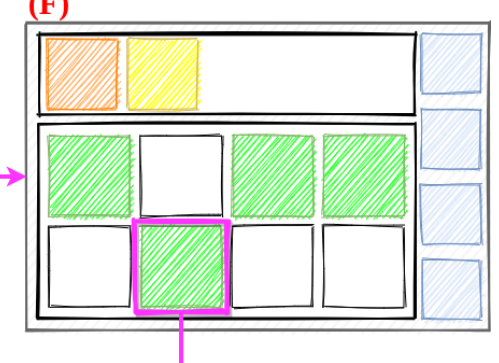

(I)

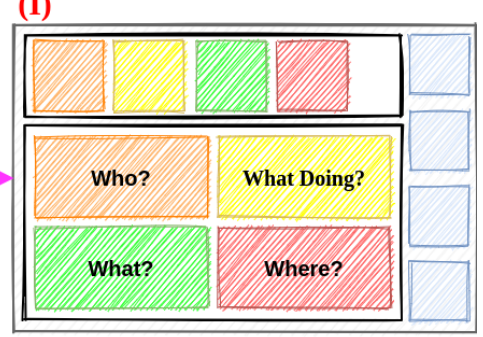

Figure 4. Sentence Construction Procedure.

\subsection{Semantic Assistive Communication Board}

We present a semantic ACB as a communication board that guides sentence construction employing a semantic script. It considers that message authoring is the essential task in augmentative and alternative communication using ACBs. Besides, people with CCN may require as much support as is available for facilitating communication [Franco et al. 2018]. In this context, the semantic script used is the Colourful Semantics (CS) therapeutic tool [Bryan 1997], which was used as a basis for constructing the semantic grammar (SG) we proposed in [Pereira et al. 2020]. This way, the SG acts as a computational resource that makes possible the implementation of the CS script in an ACB. The LAMP framework acts as an additional strategy to facilitate card searching during sentence construction. This section explains how these materials act in the ACB by illustrating the procedure for message authoring.

Figure 4 illustrates one of the possible ways for constructing sentences in the semantic ACB. Each frame represents an interaction between the user and the board, and the arrows indicate the system's flow to construct a sentence with all the CS semantic roles. As shown in Figure 4-A, the user can begin the sentence construction from Who?, or What doing?, and What? and Where? are not available on the first interaction. The flow illustrated in Figure 4 exemplifies the sentence construction beginning from the verb (i.e., What Doing?). In this initial interaction, the ACB show to the user all the verbs in 
the vocabulary (Figure 4-B), as no agent (Who?) was chosen. Once the verb is chosen, it influences which cards the ACB will show to the user in the next steps. For doing so, the ACB uses the semantic relationships presents in the SG. For example, if a verb that denotes the lexical concept eat.v.01 (cf. Figure 3) is chosen, only the cards that can act as an agent of the mentioned verb will be suggested to the user in the step illustrated in Figure 4-D. Notice that some of the cards in Figure 4-D are disabled. It occurs to keep the motor patterns in card selection. For example, if the cards shown on the screen can not act as an agent of the verb eat, it will not be available for selection. However, the cards are always on the same position, maintaining a consistent path for card selection (e.g., Persons $\rightarrow$ Professionals $\rightarrow$ Teacher). The frames in Figures 4-E to 4-H illustrates the process of selecting card for What? and Where?. Notice in Figure 4-I that, even when the sentence construction was initiated from the verb, it is shown in the direct order (i.e., Agent Verb Theme Location). This is important to help the user to associate the sequence of colors to the sentence structure, as suggested by the CS (cf. Section 2.1). In this example, all semantic roles of the proposed structure are used. However, other constructions can be made using less roles. For example: Who? + What Doing? + What?; Who? + What Doing?; What Doing? + What?; Who? + What Doing? + Where?. In addition, constructions that use more than one card in the roles Who? and What? are allowed, which enables the authoring of phrases like "me [AND] mommy eat popcorn" or "I like English [AND] math". Yet another option is to insert more semantic roles into the CS framework, as suggested by [Bolderson et al. 2011], who inserted the roles How? (i.e., manner), For whom? (i.e., recipient) and When? (i.e., time). However, this depends on the existence of such relationships in the SG.

\subsection{Technology Acceptance Evaluation}

The Technology Acceptance Model (TAM) [Davis 1985] provides explanations of factors that are considered determinants in the acceptance of a given technology [Davis et al. 1989]. TAM postulates that the acceptance of a given technology is explained by five essential factors [Davis et al. 1989]: 1) Attitude to use (ATT) - an individual's evaluative judgment on target behavior in some dimension (e.g., good/bad, harmful/beneficial); 2) Behavioral Intention to Use (BI) - the motivation or willingness of an individual to strive to perform the desired behavior; 3) Perceived Usefulness (PU) - an individual's perception that the use of particular technology will increase work performance; 4) Perceived Ease of Use (PEOU) - an individual's perception that the use of a given technology will be effortless; and 5) Actual use (AU) - an assessment of how many people use the technology provided. Generally, the AU factor is difficult to measure [Holden and Karsh 2010, Chau and Hu 2002b, Chau and Hu 2002a], especially when the technology studied is not yet in use. Thus, since BI is its closest antecedent, it is the one that best describes what acceptance is. Also, according to [Davis and Venkatesh 1996], based on empirical evidence, ATT can be excluded from the model because it cannot fully measure the effect of PU on BI. Therefore, we consider that the three essential factors for TAM's application are PU, PEOU, and BI. According to TAM, PEOU and PU are the two fundamental determinants of user acceptance of the technology. When users find technology easy to use, they perceive the technology as a useful one. Moreover, As shown in Figure 5, TAM offers the causal relationships of these two fundamental constructs (PEOU and PU) with BI. In doing so, TAM establishes that technology acceptance can be predicted by testing the following hypothesis: 


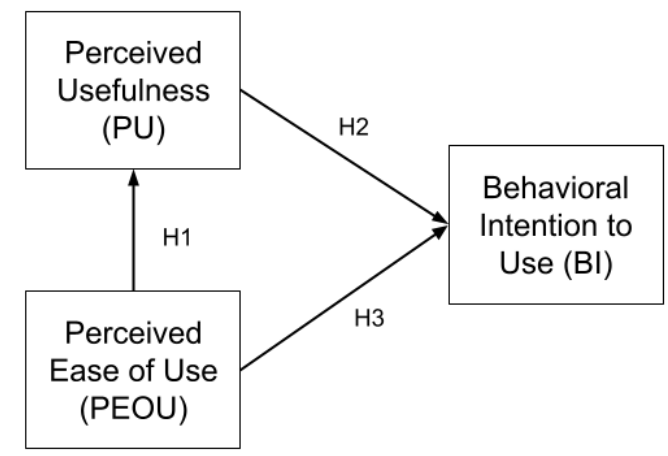

Figure 5. Simplified Technology Acceptance Model

- H1: Perceived Ease of Use has a direct effect on Perceived Usefulness.

- H2: Perceived Usefulness has a direct effect on Behavioral Intention to Use.

- H3: Perceived Ease of Use has a direct effect on Behavioral Intention to Use.

Hence, TAM's theory provides a structural model in which Independent Variables (i.e., PEOU and PU) affect a Dependent Variable (i.e., BI). This effect may be assessed by multiple regression, variance and covariance analysis, multiple discriminant analysis, or principal component analysis [Chin et al. 1998]. However, the most used methods for assessing structural models like TAM are based on Structural Equation Modelling (SEM). According to [Ullman and Bentler 2003], SEM is a collection of statistical techniques that allow a set of relationships between one or more independent variables, either continuous or discrete, and one or more dependent variables, either continuous or discrete, to be examined. In general, SEM approaches are closely identified with the Maximum Likelihood (ML) covariance structure analysis [Fornell and Bookstein 1982]. ML was generalized by [Jöreskog 1970], and it is implemented in the software LISREL [Jöreskog and Sörbom 1996]. This approach attempts to minimize the difference between the sample covariances and those predicted by the theoretical model. However, it assumes that the observed variables follow a specific multivariate distribution (i.e., normality) and that observations are independent [Fornell and Bookstein 1982]. These assumptions may not be satisfied by exploratory studies which have small sample sizes.

The Partial Least Squares-Structural Equation Modelling (PLS-SEM) [Wold 1982, Falk and Miller 1992, Thompson et al. 1995, Chin et al. 1998], emerges as an alternative for SEM analysis. Unlike covariance-based SEM approaches like LISREL, the component-based PLS uses a least-squares estimation procedure. In doing so, PLS avoids many of the restrictive assumptions underlying covariance-based SEM techniques such as multivariate normality and large sample size [Fornell and Bookstein 1982, Falk and Miller 1992]. Therefore, PLSSEM becomes a good alternative to SEM when the following situations occur [Bacon 1999, Hwang et al. 2010, Wong 2010]: 1) Sample size is small; 2) Applications have little available theory; 3) Predictive accuracy is paramount; and 4) Correct model specification cannot be ensured.

\section{Results}

The prototypical semantic ACB was presented through a video to educators and therapists of children with complex communication to assess their acceptance of using it as a ther- 
Table 1. Reliability and Validity Assessment

\begin{tabular}{lllllll}
\hline Factor & Items & Mean & Std & $\begin{array}{l}\text { Factor Load- } \\
\text { ing }\end{array}$ & $\begin{array}{l}\text { Composite } \\
\text { Reliability }\end{array}$ & AVE \\
& BI1 & 0.927 & 0.043 & 0.934 & & \\
Behavioural Intention to Use & BI2 & 0.963 & 0.024 & 0.967 & & \\
& BI3 & 0.910 & 0.047 & 0.920 & 0.973 & 0.88 \\
& BI4 & 0.966 & 0.018 & 0.967 & & \\
& BI5 & 0.876 & 0.084 & 0.901 & & \\
Perceived Ease of Use & PEOU1 & 0.747 & 0.255 & 0.832 & & \\
& PEOU2 & 0.953 & 0.037 & 0.958 & 0.931 & \\
& PEOU3 & 0.911 & 0.074 & 0.922 & & \\
& PU1 & 0.947 & 0.048 & 0.949 & & \\
Perceived Usefulness & PU2 & 0.872 & 0.060 & 0.876 & & \\
& PU3 & 0.915 & 0.043 & 0.919 & & \\
& PU4 & 0.900 & 0.035 & 0.901 & & \\
\hline
\end{tabular}

apeutic or educational tool. The participants were asked to answer a questionnaire with 15 assertive questions with a 7-point Likert scale. The questions cover the three factors considered essential for the application of TAM (cf. Section 2.5) : 1) Behavioral Intention to Use (BI) ; 2) Perceived Usefulness (PU) ; and 3) Perceived Ease of Use (PEOU). We posted the questionnaire online in social networking groups focused on the use of ACB. 34 health and education professionals from five activity areas participated in the survey. They are nineteen speech therapists, seven occupational therapists, five psychologists, two pedagogues. Regarding the experience in using ACBs, fifteen participants declared that they used it for less than two years, six between 2 and 4 years, eight between 5 and 7 years, and 12 declared that they used it for more than seven years. Considering the small sample size, we used PLS-SEM to assess the collected data. The next sub-sections present the instrument validity and reliability assessment and the structural model assessment.

\subsection{Validity and Reliability Assessment}

For verifying the validity and reliability of the measures, we observed the factor loadings (i.e., links between measures and factors) assessing the measurement model in SmartPLS 2.0 [Ringle et al. 2015]. Reliabilities of individual items were assessed by examining the loadings of the items on their respective factors. As argued by [Rivard and Huff 1988], these loadings values should be higher than 0.5 to indicate that a significant variance was shared between each item and its respective factor. Items validity was also assessed by the examination of factor loading. For those, [Peterson 2000], suggests a threshold of 0.5. As shown in Table 1, all items (e.g., BI1, BI2, and BI3) exceed both thresholds.

Factor reliability and convergent validity were also assessed by analysing Composite Reliability (CR) and Average Variance Extracted (AVE), respectively. According to [Fornell and Larcker 1981], CR is an "indicator of the shared variance among the observed variables used as an indicator of a latent construct". Our CR results vary from 0.867 to 0.973 , and thus, all are above the minimum value of 0.7 , suggested by [Nunnally and Bernstein 1994]. AVE, in turn, is defined as the grand mean value of the squared loading of items related to the factor and the common measure for establishing the convergent validity. [Hair Jr et al. 2016] argued that a value higher than 0.5 for the AVE suggests that the factor elucidates more than half of the variance of its items. As 
Table 2. Fornell-Larcker Criterion Results

\begin{tabular}{llll} 
& BI & PEOU & PU \\
\hline BI & $\mathbf{0 . 9 3 8}$ & & \\
PEOU & 0.156 & $\mathbf{0 . 9 0 5}$ & \\
PU & $0 . .525$ & 0.733 & $\mathbf{0 . 9 1 1}$ \\
\hline
\end{tabular}

shown in Table 1, all factors reached a sufficient AVE value.

The discriminant validity analysis is based on Fornell-Larcker criterion [Fornell and Bookstein 1982], and on items cross-loading analysis. Concerning the Fornell-Larcker criterion, the square root of AVE for each factor should exceed the correlation of latent variables. As shown in Table 2, all constructs satisfactorily pass the test, as the square root of the AVE (bold on the diagonal) is larger than the cross-correlations with other factors (vertical and horizontal). Regarding cross-loading, the loading of each indicator should be higher than the loadings of its corresponding variables' indicators. The results of this analysis are shown in Table 3.

\subsection{Structural Model Assessment}

Figure 6 shows the assessed model with path coefficients and $R^{2}$ values, which, according to [Hair Jr et al. 2016] are the essential measures for assessing a structural model. The $R^{2}$ value (a.k.a. coefficient of determination) is the proportion of variance in the dependent variable that the independent variables can explain. As shown, the model explains $38.7 \%$ of the variance in BI, and 53,8\% in PU. In terms of path analysis, Figure 6 demonstrates the path coefficients and $p$-values for each hypothesis, as well as the $t$-values. The $p$ values were produced with a bootstrapping procedure of 1000 resamples. As can be seen, hypotheses $\mathrm{H} 1$ and $\mathrm{H} 2$ were supported. It demonstrates that PEOU has a direct and significant effect on PU and PU in BI. However, Perceived Ease of Use had a negative effect in Behavioral Intention to Use (-0.388).

\section{Conclusions}

In this paper, we report how we combined three theories for constructing a semantic assistive communication board (ACB). The proposed ACB is based on the Colourful Seman-

Table 3. Cross Loading Results

\begin{tabular}{llll}
\hline & BI & PEOU & PU \\
\hline BI1 & $\mathbf{0 , 9 3 4}$ & 0,131 & 0,532 \\
BI2 & $\mathbf{0 , 9 6 7}$ & 0,166 & 0,533 \\
BI3 & $\mathbf{0 , 9 2 0}$ & 0,153 & 0,429 \\
BI4 & $\mathbf{0 , 9 6 7}$ & 0,139 & 0,516 \\
BI5 & $\mathbf{0 , 9 0 1}$ & 0,148 & 0,433 \\
PEOU1 & 0,136 & $\mathbf{0 , 8 3 2}$ & 0,566 \\
PEOU3 & 0,165 & $\mathbf{0 , 9 5 8}$ & 0,742 \\
PEOU5 & 0,123 & $\mathbf{0 , 9 2 2}$ & 0,669 \\
PU1 & 0,384 & 0,686 & $\mathbf{0 , 9 4 9}$ \\
PU2 & 0,410 & 0,568 & $\mathbf{0 , 8 7 6}$ \\
PU3 & 0,394 & 0,758 & $\mathbf{0 , 9 1 9}$ \\
PU4 & 0,686 & 0,648 & $\mathbf{0 , 9 0 1}$ \\
\hline
\end{tabular}




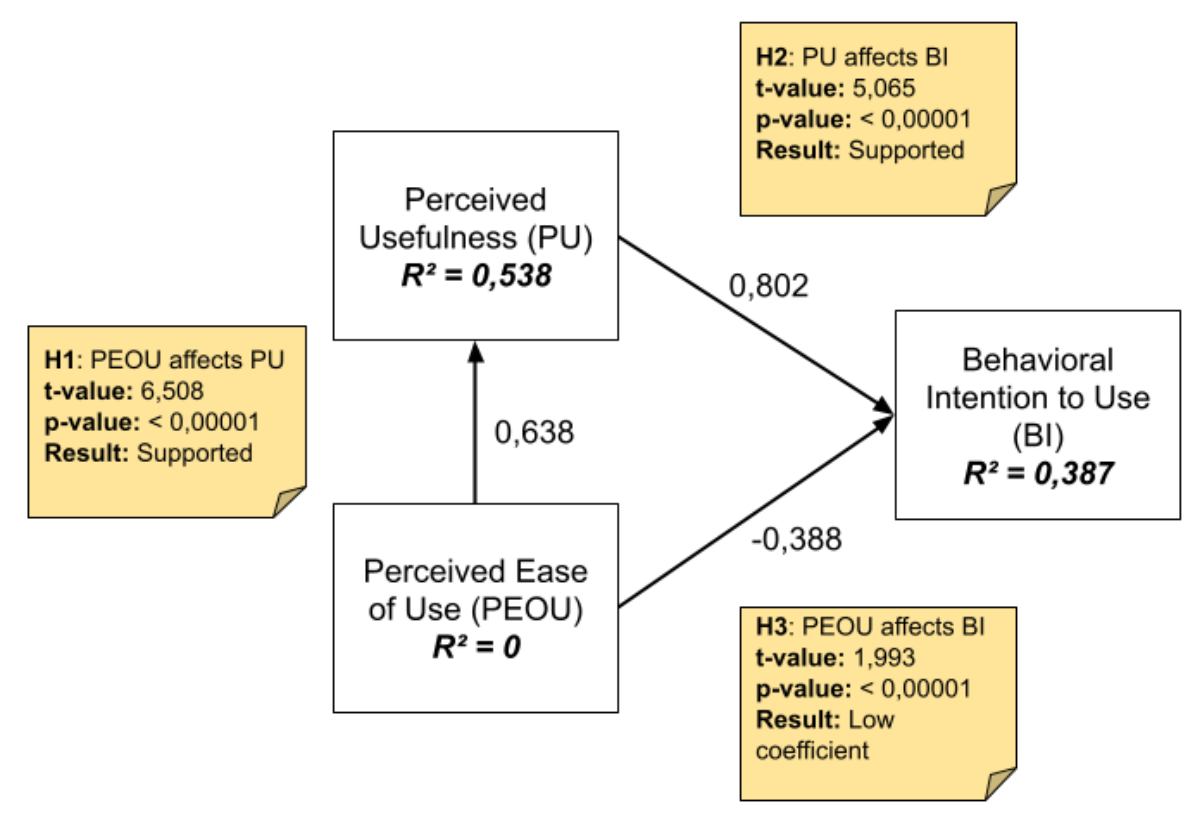

Figure 6. Structural Model Assessment and Hypothesis Testing Results

tics, a therapeutic tool used to teach children with complex communication needs (CCN) to construct and understand meaningful sentences. We used the Technology Acceptance Model (TAM) to evaluate the acceptance of health and education professionals on using the proposed $\mathrm{ACB}$ as a therapeutic tool for children with $\mathrm{CCN}$. The results demonstrate that the professionals are more interested in a useful tool than an easy to use. However, the results also suggest that the ACB's ease of use affects the professionals' perception of its usefulness. It demonstrates that the participants consider the ACB useful, but it needs to be easier to use, considering that the potential users (children with $\mathrm{CCN}$ ) may have cognitive delays. It is necessary to consider the small sample size of this exploratory research as a limitation.

The proposed ACB can be used in communication settings in which the user is familiarized with telegraphic messages. Besides, it can be used as an educational tool for teaching children with typical development or not to understand and construct wellstructured sentences. As future work, we intend to make available a tool for parents and caregivers to customize semantic ACBs according to users' needs and investigate how it can improve CCN individuals' communication. Besides, we intend to investigate ways to make the ACB easier to use and investigate its usage for therapy by means of singlesubject experiments.

\section{Acknowledgement}

This research was supported by the Coordenação de Aperfeiçoamento de Pessoal de Nível Superior - Brasil (CAPES). Grant code: [88887.481522/2020-00]. The pictographic symbols used in Figure 1 are the property of the Government of Aragón and have been created by Sergio Palao for ARASAAC (http://www.arasaac.org), that distributes them under Creative Commons License BY-NC-SA. 


\section{References}

ASHA (2021). Augmentative and alternative communication. https: //www.asha.org/practice-portal/professional-issues/ augmentative-and-alternative-communication/. September, 2021.

Bacon, L. D. (1999). Using lisrel and pls to measure customer satisfaction. In Sawtooth Software Conference Proceedings, pages 2-5.

Bedwani, M.-A. N., Bruck, S., and Costley, D. (2015). Augmentative and alternative communication for children with autism spectrum disorder: An evidence-based evaluation of the language acquisition through motor planning (lamp) programme. Cogent Education, 2(1):1045807.

Bolderson, S., Dosanjh, C., Milligan, C., Pring, T., and Chiat, S. (2011). Colourful semantics: A clinical investigation. Child Language Teaching and Therapy, 27(3):344-353.

Bryan, A. (1997). Colourful semantics: Thematic role therapy. Language disorders in children and adults: Psycholinguistic approaches to therapy, pages 143-161.

Burton, R. R. (1976). Semantic grammar: An engineering technique for constructing natural language understanding systems.

Chau, P. Y. and Hu, P. J. (2002a). Examining a model of information technology acceptance by individual professionals: An exploratory study. Journal of management information systems, 18(4):191-229.

Chau, P. Y. and Hu, P. J.-H. (2002b). Investigating healthcare professionals' decisions to accept telemedicine technology: an empirical test of competing theories. Information \& management, 39(4):297-311.

Chin, W. W. et al. (1998). The partial least squares approach to structural equation modeling. Modern methods for business research, 295(2):295-336.

Christopoulou, M., Voniati, L., Drosos, K., and Armostis, S. (2021). Colorful semantics in cypriot-greek-speaking children with autism spectrum disorder. Folia Phoniatrica et Logopaedica, 73(3):185-194.

Davis, F. D. (1985). A technology acceptance model for empirically testing new enduser information systems: Theory and results. $\mathrm{PhD}$ thesis, Massachusetts Institute of Technology.

Davis, F. D., Bagozzi, R. P., and Warshaw, P. R. (1989). User acceptance of computer technology: a comparison of two theoretical models. Management science, 35(8):9821003.

Davis, F. D. and Venkatesh, V. (1996). A critical assessment of potential measurement biases in the technology acceptance model: three experiments. International journal of human-computer studies, 45(1):19-45.

Dijksterhuis, A., Aarts, H., Bargh, J. A., and Van Knippenberg, A. (2000). On the relation between associative strength and automatic behavior. Journal of Experimental Social Psychology, 36(5):531-544.

Falk, R. F. and Miller, N. B. (1992). A primer for soft modeling. University of Akron Press. 
Fornell, C. and Bookstein, F. L. (1982). Two structural equation models: Lisrel and pls applied to consumer exit-voice theory. Journal of Marketing research, 19(4):440-452.

Fornell, C. and Larcker, D. F. (1981). Evaluating structural equation models with unobservable variables and measurement error. Journal of marketing research, 18(1):39-50.

Franco, N., Silva, E., Lima, R., and Fidalgo, R. (2018). Towards a reference architecture for augmentative and alternative communication systems. In Brazilian Symposium on Computers in Education (Simpósio Brasileiro de Informática na Educação-SBIE), volume 29, page 1073.

Ganz, J. B., Boles, M. B., Goodwyn, F. D., and Flores, M. M. (2014). Efficacy of handheld electronic visual supports to enhance vocabulary in children with asd. Focus on Autism and Other Developmental Disabilities, 29(1):3-12.

Ganz, J. B. and Hong, E. R. (2014). Naturalistic aided aac instruction. In Aided augmentative communication for individuals with autism spectrum disorders, pages 55-75. Springer.

Hair Jr, J. F., Hult, G. T. M., Ringle, C., and Sarstedt, M. (2016). A primer on partial least squares structural equation modeling (PLS-SEM). Sage publications.

Halloran, J. and Halloran, C. (2006). Lamp: Language acquisition through motor planning. Wooster $(\mathrm{OH})$ : Prentke Romich Company.

Hernández, S. S., Mancilla, D., Medina, J. M., and Iregui, M. (2014). User-centric recommendation model for aac based on multi-criteria planning. In International Conference on Advances in Human-oriented and Personalized Mechanisms, Technologies and Services.

Holden, R. J. and Karsh, B.-T. (2010). The technology acceptance model: its past and its future in health care. Journal of biomedical informatics, 43(1):159-172.

Hwang, H., Malhotra, N. K., Kim, Y., Tomiuk, M. A., and Hong, S. (2010). A comparative study on parameter recovery of three approaches to structural equation modeling. Journal of Marketing Research, 47(4):699-712.

Jöreskog, K. G. (1970). A general method for estimating a linear structural equation system. ETS Research Bulletin Series, 1970(2):i-41.

Jöreskog, K. G. and Sörbom, D. (1996). LISREL 8: User's reference guide. Scientific Software International.

Koegel, L. K., Koegel, R. L., Harrower, J. K., and Carter, C. M. (1999). Pivotal response intervention i: Overview of approach. Journal of the Association for Persons with Severe Handicaps, 24(3):174-185.

Koegel, R. L., Koegel, L. K., and Brookman, L. I. (2003). Empirically supported pivotal response interventions for children with autism. Evidence-based psychotherapies for children and adolescents, 341357.

Law, J., Lee, W., Roulstone, S., Wren, Y., Zeng, B., and Lindsay, G. (2012). 'what works': interventions for children and young people with speech, language and communication needs. 
Nunnally, J. C. and Bernstein, I. H. (1994). Psychometric theory. Tata McGraw-Hill Education.

Pereira, J., Franco, N., and Fidalgo, R. (2020). A semantic grammar for augmentative and alternative communication systems. In Sojka, P., Kopeček, I., Pala, K., and Horák, A., editors, Text, Speech, and Dialogue, pages 257-264, Cham. Springer International Publishing.

Peterson, R. A. (2000). A meta-analysis of variance accounted for and factor loadings in exploratory factor analysis. Marketing Letters, 11(3):261-275.

Ringle, C., Da Silva, D., and Bido, D. (2015). Structural equation modeling with the smartpls. Bido, D., da Silva, D., \& Ringle, C.(2014). Structural Equation Modeling with the Smartpls. Brazilian Journal Of Marketing, 13(2).

Rivard, S. and Huff, S. L. (1988). Factors of success for end-user computing. Communications of the ACM, 31(5):552-561.

Salmoni, A. W., Schmidt, R. A., and Walter, C. B. (1984). Knowledge of results and motor learning: a review and critical reappraisal. Psychological bulletin, 95(3):355.

Thompson, R., Barclay, D., and Higgins, C. A. (1995). The partial least squares approach to causal modeling: Personal computer adoption and use as an illustration. Technology studies: special issue on research methodology, 2(2):284-324.

Ullman, J. B. and Bentler, P. M. (2003). Structural equation modeling. Handbook of psychology, pages 607-634.

Wold, H. (1982). Systems under indirect observation using pls. A second generation of multivariate analysis: Methods.

Wong, K. (2010). Handling small survey sample size and skewed dataset with partial least square path modelling. Vue: the magazine of the Marketing Research and Intelligence Association, 20:20-23. 